\title{
Líquido amniótico tópico: uma potencial nova alternativa para doenças da superfície ocular
}

\section{Topical amniotic fluid: a potential new alternative for ocular surface diseases}

\author{
Guilherme Goulart Quinto $^{1}$ \\ Suy Anne Rebouças Martins ${ }^{2}$ \\ Renata Tiemi ${ }^{3}$ \\ MauroCampos ${ }^{4}$ \\ Ashley Behrens ${ }^{5}$
}

\section{RESUMO}

O líquido amniótico banha o feto durante a vida intra-uterina e está em contato permanente com a superfície ocular durante este importante período do desenvolvimento. Ele contém uma série de fatores de crescimento que podem ter diversos efeitos sobre o processo cicatricial. Estes fatores aceleram a recuperação da sensibilidade corneana e regeneração nervosa após procedimentos cerato-refrativos, além de controlar a formação de cicatriz e o equilíbrio da superfície ocular após sua aplicação tópica. Centenas de diferentes proteínas têm sido identificadas no líquido amniótico humano e o papel de cada uma continua desconhecido. Os resultados obtidos até o momento sobre a aplicação de líquido amniótico em doenças de superfície ocular sugerem uma terapia promissora. Pesquisas estão sendo realizadas para identificar os efeitos dos fatores específicos do líquido amniótico sobre a inflamação ocular. $\mathrm{O}$ objetivo desta revisão é relatar as propriedades e utilizações atuais do líquido amniótico, bem como apresentar os recentes estudos relacionados ao uso deste líquido e doenças da superfície ocular.

Descritores: Líquido amniótico; Soluções oftálmicas/uso terapêutico; Cicatrização de feridas; Doenças da córnea/terapia

Trabalho realizado no Wilmer Ophthalmological Institute - Baltimore (MD) - USA.

${ }^{1}$ Oftalmologista, Fellow em Córnea e Cirurgia Refrativa - Wilmer Ophthalmological Institute, The Johns Hopkins University School of Medicine - Baltimore (MD) - USA. Pós-graduando em nível doutorado pela Universidade Federal de São Paulo - UNIFESP - São Paulo (SP) - Brasil.

${ }^{2}$ Fellow em Córnea e Cirurgia Refrativa - Wilmer Ophthalmological Institute, The Johns Hopkins University School of Medicine - Baltimore (MD) - USA. Doutora em Oftalmologia pela Universidade Federal de São Paulo - UNIFESP - São Paulo (SP) - Brasil.

${ }^{3}$ Oftalmologista, Pós-graduanda em nível doutorado pela Universidade Federal de São Paulo - UNIFESP - São Paulo (SP) - Brasil.

${ }^{4}$ Professor Adjunto Livre-Docente do Departamento de Oftalmologia da UNIFESP - São Paulo (SP) - Brasil.

${ }^{5}$ Professor do Wilmer Ophthalmological Institute, The Johns Hopkins University School of Medicine Baltimore (MD) - USA.

Os autores não possuem interesse comercial ou financeiro em nenhum produto mencionado no presente manuscrito.

Endereço para correspondência: Ashley Behrens, M.D. The Wilmer Eye Institute 600 North Wolfe St, 255 Woods Building - Baltimore, MD 21287-0005, USA. E-mail: abehrens@jhmi.edu

\section{INTRODUÇÃO}

A membrana amniótica humana (MAH) é um tecido biológico complexo que possui proteínas antiangiogênicas e antiinflamatórias ${ }^{(1)}$, e tem demonstrado efeitos benéficos em limitar o dano ocular após injúrias à superfície ocular ${ }^{(2-4)}$. O efeito positivo sobre a reepitelização corneana, resposta inflamatória e formação de cicatriz têm sido relatados com o uso de $\mathrm{MAH}^{(5-6)}$. Em estudo prospectivo randomizado de córneas de coelhos submetidas à ceratectomia fotorrefrativa com "excimer laser", alguns autores demonstraram a redução de "haze" durante o período pós-operatório após a aplicação de membrana amniótica na área ablada, e eles sugerem que isso se deva provavelmente à redução da infiltração de células inflamatórias que ocorrem no período pós-operatório precoce ${ }^{(7)}$. Além disso, outros autores criaram um defeito epitelial em córneas de coelhos utilizando "excimer laser", e relataram uma rápida taxa de reepitelização quando as córneas eram tratadas com membrana amniótica ${ }^{(8)}$.

In vivo, a MAH é banhada pelo líquido amniótico humano (LAH) e ambos contêm componentes potencialmente terapêuticos ${ }^{(9)}$. Durante o desenvolvimento embrionário, o LAH se encontra em contato com a superfície ocular e é capaz de modular o processo de cicatrização no feto ${ }^{(10-11)}$. Tem sido relatado que não existe formação de cicatriz ou reação inflamatória sig-
\end{abstract}


nificativa no processo de cicatrização da pele na vida intrauterina, entretanto os resultados destes estudos continuam controversos ${ }^{(12)}$. Tem-se demonstrado que o LAH é efetivo na redução de opacidade de córnea e formação de cicatriz de córnea em camundongos após indução de queimadura por álcalis ${ }^{(13)}$. Além disso, relatos indicam que o LAH acelera a recuperação da sensibilidade corneana através da regeneração nervosa, assim como diminui a formação de cicatriz após ablação com "excimer laser" em coelhos ${ }^{(14)}$. Com base nessas observações, espera-se que a MAH e o LAH possuam efeitos terapêuticos similares. $\mathrm{O}$ presente texto tem o propósito de revisar as propriedades e as potenciais utilizações do líquido amniótico, bem como relatar as contribuições recentes relacionadas à superfície ocular.

\section{Características e propriedades do líquido amniótico}

O líquido amniótico é um complexo e dinâmico líquido biológico que proporciona proteção mecânica, nutrientes e substâncias necessárias para o crescimento fetal e seu bemestar, além de possuir efeito antimicrobiano. Ele se encontra em contato permanente com o feto e sua composição se altera de acordo com evolução gestacional ${ }^{(15)}$. Durante a embriogênese, o LAH é inicialmente proveniente do plasma materno, o qual atravessa as membranas fetais. Devido à difusão livre que ocorre bidirecionalmente entre o LAH e o feto, através da pele fetal, placenta e cordão umbilical de 10 a 20 semanas de gestação, a composição do LAH se torna semelhante ao do plasma do feto durante esse período ${ }^{(16-17)}$.

O LAH é composto por 98 a $99 \%$ de água, sendo que o restante de sua composição é constituída por sais orgânicos e inorgânicos, assim como células fetais descamadas. Os constituintes orgânicos são compostos por proteínas, peptídeos, carboidratos, lipídeos, enzimas, piruvato, lactato, hormônios e eletrólitos ${ }^{(15)}$.

Os líquidos biológicos complexos, como o soro humano e o LAH, contêm diversas proteínas. O LAH possui pelo menos 842 proteínas identificadas recentemente, e o papel de cada uma ainda permanece desconhecido. Na tabela 1 se encontram as 15 proteínas presentes no LAH em maior quantidade. A albumina representa cerca de $50 \%$ e $70 \%$ da composição do plasma e do LAH, respectivamente. Apenas 36\% das proteínas presentes no LAH também fazem parte do plasma, ou seja, o LAH possui uma composição bastante diferente do plasma, o que lhe confere diversas funções. Algumas dessas funções das proteínas incluem estimulação da motilidade celular, desenvolvimento e função de órgãos, regulação do crescimento e proliferação celular, apoptose e ciclo celular, substrato para a interação celular, desenvolvimento do sistema hematológico e resposta imune ${ }^{(16)}$.

O LAH contém fatores de crescimento tais como o fator de crescimento epidérmico (EGF), o fator de crescimento insulina tipo I (IGF-I $)^{(15)}$, o fator de crescimento transformador $\alpha$ (TGF- $\alpha$ ), $\mathrm{o}$ fator de crescimento transformador $\beta-1$ (TGF- $\beta 1)^{(18)}$ e o fator de crescimento neural (NGF) ${ }^{(19)}$.
Várias citoquinas (interleucinas, IL) têm sido detectadas na MAH e no líquido amniótico: IL-6, IL-8, IL-1 $\alpha$, IL-1 $\beta$, IL-1 receptor agonista e $\mathrm{IL}-10^{(20)}$. As duas últimas têm sido demonstradas gerar significante atividade antiinflamatória ${ }^{(21)}$. Entretanto, a real interação entre todas essas proteínas, ou efeito benéfico potencial, permanecem desconhecidos.

Durante o segundo trimestre de gestação, o LAH contém altas concentrações de ácido hialurônico (AH) e estimulador da ativação de ácido hialurônico ${ }^{(22)}$. O AH, um polissacarídeo linear de alto peso molecular, é conhecido por reduzir a formação de cicatriz por inibir a migração linfocítica, a proliferação e a quimiotaxia, a fagocitose granulocítica e a degranulação, além da motilidade do macrófago ${ }^{(14)}$. O AH atua na diferenciação celular, na motilidade e na aderência celular, e tem sido sugerido ter efeitos antiinflamatórios ${ }^{(11)}$. Além disso, possui a capacidade de aumentar a regeneração tecidual através da retenção de grandes quantidades de água ${ }^{(22)}$.

Pode-se citar como substâncias com ação antimicrobiana que participam do sistema imune inato e são encontradas no LAH: as defensinas- $\alpha$, a lactoferrina, a lisozima, a calprotectina, a psoriasina e a protease inibidora da secreção de leucócitos. Todas essas substâncias apresentam atividade de amplo espectro contra bactérias, fungos, protozoários e vírus ${ }^{(15)}$.

\section{O líquido amniótico e o processo cicatricial}

Tem sido relatado que o processo de cicatrização fetal ocorre rapidamente e sem formação de cicatriz, fibrose ou inflamação ${ }^{(12)}$. Parece ser um um processo diferente do adulto ${ }^{(11)}$. A resposta do adulto à injúria tecidual inclui as fases de inflamação, proliferação e remodelação bem caracterizadas, enquanto o mecanismo envolvido no reparo tecidual fetal ainda é desconhecido ${ }^{(23)}$. O LAH contém fatores que parecem minimizar a cicatriz ${ }^{(24)}$. Incisões fetais realizadas no início da gestação irão se regenerar sem deixar cicatriz, enquanto incisões no final da gestação evoluem com formação de cicatriz. Há duas teorias para explicar esse achado. A primeira, que o AH inibiria a síntese de colágeno. Este ambiente rico em AH é devido à falta relativa de hialuronidase no LAH e à presença de estimulador da ativação de $\mathrm{AH}$ no $\mathrm{LAH}^{(23)}$. Em um estudo que avaliou o efeito do LAH sobre as proteases importantes para o processo de cicatrização, foi demonstrado que o LAH aumentou a atividade das colagenases, mas não inibiu a atividade da hialuronidase, da elastase e da cathepsin $^{(25)}$. A segunda teoria sugere que o TGF- $\beta$, o qual está ausente no LAH no início da gestação, mas presente no término da gestação, participaria ativamente da formação de cicatriz ${ }^{(26)}$. Não está claramente definido se a cicatrização ocorre sem formação de cicatriz no início da gestação, devido ao ambiente fetal favorável, como o soro fetal e LAH, ou por causa das propriedades da pele fetal.

Alguns autores avaliaram os efeitos do LAH sobre nervos periféricos com dano e a sua regeneração em modelo animal(24). Quarenta ratos tiveram seus nervos ciáticos seccionados e raparados com sutura e divididos em dois grupos de acordo com a solução aplicada: $0,3 \mathrm{ml}$ LAH no grupo experi- 


\begin{tabular}{|c|c|c|c|c|}
\hline \multicolumn{2}{|c|}{ Proteoma do LA } & \multirow{2}{*}{$\begin{array}{c}\text { Posição } \\
\text { em quantidade }\end{array}$} & \multicolumn{2}{|c|}{ Proteoma do plasma } \\
\hline Gene & Proteína & & Gene & Proteína \\
\hline ALB & Albumina & 1 & ALB & Albumina \\
\hline Igs & Imunoglobulinas & 2 & Igs & Imunoglobulinas \\
\hline FN1 & Fibronectina & 3 & TF & Serotransferrina \\
\hline TF & Serotransferrina & 4 & $\mathrm{FG}$ & Fibrinogênio \\
\hline C3 & Complemento $\mathrm{C} 3$ & 5 & AMBP & $\alpha 1$-Microglobulina \\
\hline SERPINA1 & $\alpha 1$-Antitripsina & 6 & SERPINA & $\alpha 1$-Antitripsina \\
\hline $\mathrm{CP}$ & Ceruloplasmina & 7 & C3 & Complemento $\mathrm{C} 3$ \\
\hline AFP & $\alpha$-Fetoproteína & 8 & $\mathrm{HP}$ & Haptoglobulina \\
\hline $\mathrm{GC}$ & Vitamina D & 9 & APOA1 & Apolipoproteína A-I \\
\hline POSTN & Periostina & 10 & APOB & Apolipoproteina B \\
\hline APOA1 & Apolipoproteína A-I & 11 & ORM1 & $\alpha 1$-Glicoproteína ácida \\
\hline SERPINC1 & Antitrombina III & 12 & & Lipoproteína \\
\hline TGFBI & $\begin{array}{l}\text { Precursor induzido da } \\
\text { proteína Ig-h3 do fator de } \\
\text { crescimento transformador } \beta\end{array}$ & 13 & $\mathrm{CFH}$ & Fator $\mathrm{H}$ \\
\hline AMBP & $\alpha 1$-Microglobulina & 14 & $\mathrm{CP}$ & Ceruloplasmina \\
\hline PLG & Plasminogênio & 15 & $\mathrm{C} 4$ & Complemento C4 \\
\hline
\end{tabular}

mental e $0,3 \mathrm{ml}$ de solução salina no grupo controle. Os ratos tratados com LAH, após quatro semanas do procedimento, demonstraram uma significativa redução na quantidade de tecido com cicatriz ao redor da área do procedimento $(p<0,05)$. A recuperação funcional dos nervos tratados com LAH se deu de forma mais rápida do que no grupo controle $(\mathrm{p}<0,05)$. Os autores concluíram que o LAH aumentava a regeneração nervosa periférica.

Karaçal et al., criaram defeitos ósseos em coelhos e os dividiram em dois grupos. O grupo 1, no momento da criação do defeito, recebeu instilação de LAH, enquanto no grupo 2 instilou-se apenas solução salina ${ }^{(22)}$. O grupo 1 mostrou significativo aumento da ossificação quando comparado ao grupo 2. Ao estudo histológico, seis semanas após o procedimento, os defeitos tratados com LAH tiveram ossificação superior ao grupo controle. Devido ao seu efeito positivo no processo de cicatrização óssea, e também pela facilidade de ser armazenado no congelador, o LAH parece ser útil tratamento adjunto no processo de cicatrização óssea.

Um grupo de autores relataram os efeitos do LAH sobre a cicatrização de fraturas da tíbia realizados em ratos, os quais foram divididos em três grupos. $\mathrm{O}$ grupo 1 recebeu sobre as linhas de fratura a instilação de LAH coletado na $18^{\mathrm{a}}$ semana de gestação; o grupo 2 recebeu sobre as linhas de fratura a instilação de LAH coletado no final da gestação, enquanto o grupo 3 não se aplicou nenhum tratamento ${ }^{(27)}$. $\mathrm{O}$ resultado mostrou que a pontuação de cura da fratura foi maior no grupo 1 radiologicamente na terceira e quinta semanas pós-operatórios $(\mathrm{p}<0,037, \mathrm{p}=0,018$ respectivamente). Ao estudo cintilográfico, atividade metabólica na área da fratura foi observada no grupo 1 maior do que os outros grupos na terceira semana pós-operatória. Esses resultados sugerem que o LAH possui efeitos positivos sobre a fratura de tíbia neste modelo animal.
Têm sido realizados estudos em coelhos sob o efeito do LAH em cartilagens ${ }^{(28-29)}$. Os autores demonstraram que o LAH foi capaz de aumentar a formação de cartilagem a partir de enxerto pericondrial livre. O efeito preventivo do LAH na formação de cicatriz, e seu rico conteúdo em fatores de crescimento e precursores da matriz extracelular, possivelmente estejam envolvidos neste resultado.

O efeito da aplicação de LAH também tem sido relatado no processo de cicatrização de tendões em coelhos. Ozgenel et al., avaliaram macroscopicamente e histologicamente o processo cicatricial após reparo cirúrgico de tendões associado a aplicação de LAH em 32 coelhos 12 semanas após o procedimento $^{(30)}$. Tendões tratados com LAH tiveram aumento significativo na capacidade de tensão. Desta forma, a aplicação tópica de LAH imediatamente após a tenorrafia demonstrou ser significantemente efetiva em prevenir formação de adesões peri-tendíneas sem a limitação da cicatrização neste modelo animal.

\section{Utilização do líquido amniótico em doenças de superfície ocular}

Kim e Tseng ${ }^{(5)}$ demonstraram que a cicatriz corneana pode ser reduzida através da aplicação de MAH após foto-ablação da córnea e sugeriram que a interação dos fatores do MAH pode facilitar o restabelecimento do ambiente fetal para o processo de cicatrização, pela inibição da ativação dos fibroblastos e rápida cobertura epitelial. Longaker et al. ${ }^{(31)}$, relataram que o AH deve modular o processo de cicatrização por regeneração e não pela formação de cicatriz ou fibrose. Supõe$\mathrm{se}$, assim, que o $\mathrm{AH}$ potencializa o crescimento neuronal com inibição da formação de cicatriz e invasão celular inflamatória.

Em 1996, Lee e $\operatorname{Kim}^{(14)}$ avaliaram o efeito da aplicação tópica de LAH na recuperação da sensibilidade corneana, na 
regeneração nervosa e na formação de cicatriz em córneas após ceratectomia fotorrefrativa com "excimer laser" $193 \mathrm{~nm}$ (VISX 20/20, 6 mm de diâmetro, 5 Hz) em 18 coelhos. Enquanto nove coelhos foram submetidos a uma profundidade de ablação de $70 \mu \mathrm{m}$, os demais tiveram uma profundidade de ablação de $100 \mu \mathrm{m}$. Após a ablação, o LAH foi topicamente aplicado no olho direito (grupo LAH) e solução salina balanceada (BSS) foi aplicada no olho esquerdo (grupo BSS), ambos administrados cinco vezes ao dia pelo período de um mês. O LAH foi coletado através de amniocentese de mulheres em idade gestacional de 13 a 16 semanas. Após coletado, foi centrifugado a 3.000 rotações por minuto por 15 minutos, e o sobrenadante foi coletado e armazenado a $-70^{\circ} \mathrm{C}$, com intervalo entre a coleta e o uso de uma semana no máximo. A sensibilidade corneana foi medida utilizando estesiômetro Cochet-Bonnet, enquanto a regeneração corneana se monitorou através de corante de cloreto de ouro após $1^{\underline{a}}, 2^{\underline{a}}, 4^{\underline{a}}, 5^{\underline{a}}, 8^{\underline{a}}$ e $12^{\underline{a}}$ semanas. A sensibilidade corneana foi maior no grupo ablado com $70 \mu \mathrm{m}$ do que no ablado com $100 \mu \mathrm{m}$ na primeira semana pós-operatório $(p<0,01)$. A sensibilidade se recuperou em $75 \%$ no grupo LAH e $63 \%$ no grupo BSS em uma semana $(p<0,01$ e em 12 semanas, aumentou para 97\% no grupo LAH e $93 \%$ no grupo BSS $(p<0,05)$. A cicatrização do estroma superficial foi mais destacada no grupo ablado com $100 \mu \mathrm{m}$ do que no grupo ablado com $70 \mu \mathrm{m}$. No grupo de $100 \mu \mathrm{m}$, a formação de "haze" estromal e aumentada celularidade no estroma anterior foram mais marcantes no grupo BSS do que no grupo LAH. O estado de regeneração resultante do nervo corneano demonstrou estar relacionado com a opacidade corneana. Em síntese, os restabelecimentos da sensibilidade e da regeneração nervosa foram mais rápidos no grupo LAH. Estes achados sugerem que os fatores do LAH ajudaram a restabelecer a sensibilidade corneana, regeneração nervosa e reduzir a formação de "haze". Além disso, os autores postularam que o LAH do segundo trimestre poderia ser ideal para a aplicação tópica porque estaria menos contaminado pela urina do feto $\mathrm{e}$ citoquinas inflamatórias.

Em 2006, Herretes et al., avaliaram o potencial do uso tópico do LAH para tratar queimadura ocular por álcalis em 30 camundongos C57BL/6. O LAH foi obtido em dois diferentes momentos da gestação: 16 a 18 semanas (LAH pré-termo) e 36 a 38 semanas (LAH a termo) $)^{(13)}$. Após a coleta, o LAH foi centrifugado a 1.800 rotações por minuto por 10 minutos, e o sobrenadante foi preservado a $-20^{\circ} \mathrm{C}$ até o uso, aproximadamente uma semana depois de obtido. A queimadura por álcalis foi induzida no olho direito dos camundongos seguindo modelo previamente descrito. Os animais foram divididos conforme o tratamento a ser administrado: LAH pré-termo (grupo 1), LAH a termo (grupo 2), e solução salina isotônica (grupo 3). O tratamento foi aplicado cinco vezes ao dia na primeira semana, e três vezes ao dia na segunda semana após a indução do modelo. A média de defeito epitelial para o dia 2 e o dia 4 foi significativamente menor nos grupos $1(p=0,0076)$ e $2(p=0,0031)$, quando comparadas com o grupo controle, e não houve diferença estatística significativa entre os dois grupos tratados com LAH $(p=0,52)$. Ao estudo histológico, não houve diferença significativa na contagem de células inflamatórias quando comparadas com tratamento à base de LAH e olhos não queimados. Os olhos tratados com a solução salina tiveram um significativo aumento na contagem das células quando comparadas com os olhos não queimados. Neste estudo, o LAH pré-termo e o LAH a termo foram efetivos na redução da opacidade corneana, da cicatriz e da promoção da re-epitelização quando comparadas com a solução salina. Os autores tiveram evidências observacionais de que o LAH a termo deveria melhorar o resultado final quando comparado com o LAH pré-termo em todos os aspectos estudados. Estes achados, entretanto, não foram estatisticamente significativos provavelmente pelo pequeno número da amostra.

Kasper et al., realizaram um estudo in vitro comparando o líquido amniótico com soro autólogo ${ }^{(32)}$. O LAH foi obtido de 10 grávidas durante amniocentese para diagnóstico pré-natal com 14 semanas de idade gestacional, enquanto o soro autólogo foi obtido de voluntários. Tanto o LAH quanto o soro foram diluídos em solução salina balanceada em concentrações de $100 \%$, $50 \%, 25 \%, 12,5 \%, 6,25 \%$. O soro autólogo demonstrou ser melhor para suportar o crescimento celular durante o período de incubação. Tanto para o LAH quanto para o soro, o crescimento celular foi reduzindo com o aumento da diluição. Fatores de crescimento como EGF, TGF- $\beta$, e fibronectina foram encontrados em níveis mais elevados no soro. Em resumo, o soro teve resultado superior para suportar o crescimento e a migração no epitélio corneano do que o LAH neste estudo.

Castro-Combs et al., avaliaram os efeitos tópicos do líquido amniótico humano e o líquido amniótico eqüino na reepitelização e processo cicatricial estromal ${ }^{(33)}$. Em modelo ex vivo, um total de 52 córneas de coelhos foram cultivadas e divididas em quatro grupos de tratamento: soro bovino fetal (SBF), LAH, líquido amniótico eqüino (LAE), e controle (solução tampão fosfato-PBS). Com um trépano, foram marcados $8,5 \mathrm{~mm}$ de diâmetros da córnea central e esta área selecionada para desepitelização. Os defeitos epiteliais foram realizados e documentados a cada 8 horas, por 72 horas, após administração de fluoresceína. Não houve diferença significativa na velocidade de fechamento do defeito epitelial corneano entre o SBF e o grupo controle $(p>0,06)$. O defeito epitelial foi significativamente menor no grupo LAH comparado ao grupo controle após 16 horas $(p=0,008), 64$ horas $(p=0,0072)$ e 72 horas $(\mathrm{p}=0,016)$. A média da área do defeito epitelial foi significativamente menor no grupo LAE comparado ao grupo controle após 24 horas $(\mathrm{p}=0,016), 40$ horas $(\mathrm{p}=0,032), 64$ horas $(p=0,008)$ e 72 horas $(p=0,007)$. O defeito epitelial no grupo LAH e LAE foi menor do que no SBF, porém sem diferença significativa. Ao estudo histológico, a média da densidade de ceratócitos foi maior no estroma anterior nos grupos LAH $(p=0,015)$ e LAE $(p=0,016)$ quando comparados com o grupo controle. Este estudo indicou que a aplicação tópica de LAH e LAE estaria associada com acelerada reepitelização da córnea neste modelo de cultura ex vivo. A densidade de ceratócitos seria menos afetada após o dano epitelial utilizando este 
tratamento. Este efeito protetor sob a densidade dos ceratócitos supostamente decorreria da maior taxa de proliferação nos grupos tratados por LAH e LAE.

\section{Utilizações como meio de cultura para células-tronco}

No futuro, a caracterização e o isolamento de diferentes células-tronco humanas permitirão uma investigação molecular detalhada do processo de diferenciação celular, assim como o estabelecimento de novos conceitos terapêuticos para uma imensa variedade de doenças. Desde o primeiro isolamento e cultura de células-tronco embriônicas humanas há 10 anos, sua utilização em pesquisa e tratamento tem sido contestada por considerações éticas, bem como pelo risco de malignização de células-tronco indiferenciadas após transplante em humanos. A favor das células-tronco adultas identifica-se a aceitação ética e o baixo risco de desenvolvimento tumoral. Entretanto, o potencial de diferenciação e capacidade proliferativa das mesmas são limitadas ${ }^{(34)}$. Há quatro anos, a descoberta de células-tronco no LAH, expressando Oct-4, um marcador específico de célula-tronco pluripotente, iniciou uma nova e promissora área para pesquisas de células-tronco ${ }^{(35-36)}$. As células-tronco do líquido amniótico possuem o potencial para diferenciação em células das três camadas embriogênicas (ectoderma, mesoderma, endoderma) ${ }^{(37)}$. Estas células não formam tumores in vivo e não levantam problemas éticos associados com as células-tronco embriônicas humana ${ }^{(34)}$.

\section{Complicações e riscos decorrentes da utilização de material biológico}

A transmissão de doenças infecciosas é sempre um risco associado com tratamentos realizados com tecidos humanos. Potenciais doadores necessitam serem rastreados efetivamente para qualquer fator de risco que possa torná-lo incapaz para doar. Estes deverão ser submetidos a uma anamnese detalhada para assegurar ausência de fatores de risco com evidências clínicas de HIV, hepatite B, hepatite C, HTLV, citomegalovírus, sífilis, doença de Creutzfeld-Jacob e tuberculose. O consentimento do doador deve ser obtido e rastreamento para infecções deve ser realizado. O doador deve ser testado para HIV-1/2, hepatite B e hepatite C. Existe uma chance pequena de o doador estar em janela imunológica para determinada infecção. Desta forma, mesmo que os testes sorológicos sejam negativos, aconselha-se que os exames sejam repetidos após seis meses. $\mathrm{O} \mathrm{LAH}$ pode ser preservado a $-80^{\circ} \mathrm{C}$ até que ambos os testes sejam negativos. Embora não seja possível excluir todas as doenças infecciosas, pode-se reduzir drasticamente a possibilidade de um evento adverso infeccioso, no momento da preparação do tecido, mantendo-se o LAH em condições de estrita assepsia ${ }^{(38)}$.

\section{CONCLUSÃO}

O LAH é um produto biológico com propriedades antiinflamatórias, antiinfecciosas, regeneradoras e de redução da for- mação de cicatriz. Os resultados obtidos na aplicação do LAH em doenças de superfície ocular sugerem um potencial terapêutico promissor. Entretanto, são necessários estudos mais extensos que confirmem amplamente os achados identificados até o momento. A confirmação dos achados e a comprovação em humanos de que o LAH de fato possui o poder de redução da formação de cicatriz trará, indiscutivelmente, novas possibilidades terapêuticas para determinadas doenças da superfície ocular.

\section{ABSTRACT}

Amniotic fluid bathes the fetus during intrauterine life and is in permanent contact with the fetal ocular surface in this important period of development. It contains a series of growth factors that may have multiple effects on the wound healing process. These factors are thought to accelerate the recovery of corneal sensitivity and nerve regeneration after keratorefractive procedures, and also may control scar formation and balance the ocular surface after topical application. Hundreds of different proteins have been identified in the human amniotic fluid, and the role of each still not quite understood. The outcomes obtained so far with amniotic fluid application to ocular surface diseases suggest a promising therapy. Research is underway to identify the effects of specific factors of the amniotic fluid in ocular inflammation. The purpose of this review is to report the properties and current utilizations of amniotic fluid as well as to summarize the recent studies related to the use of this fluid for ocular surface diseases.

Keywords: Amniotic fluid; Ophthalmic solutions/therapeutic use; Wound healing; Corneal diseases/therapy

\section{REFERÊNCIAS}

1. Hao Y, Ma DH, Hwang DG, Kim WS, Zhang F. Identification of antiangiogenic and antiinflammatory proteins in human amniotic membrane. Cornea. 2000; 19(3):348-52.

2. Dua HS, Gomes JA, King AJ, Maharajan VS. The amniotic membrane in ophthalmology. Surv Ophthalmol. 2004;49(1):51-77.

3. Uçakhan OO, Köklü G, Firat E. Non-preserved human amniotic membrane transplantation in acute and chronic chemical eye injuries. Cornea. 2002;21(2):169-72.

4. Kim JS, Kim JC, Na BK, Jeong JM, Song CY. Amniotic membrane patching promotes healing and inhibits proteinase activity on wound healing following acute corneal alkali burn. Exp Eye Res. 2000;70(3):329-37.

5. Kim JC, Tseng SC. Transplantation of preserved human amniotic membrane for surface reconstruction in severely damaged rabbit corneas. Cornea. 1995;14(5): 473-84.

6. Meller D, Tseng SC. [Reconstruction of the conjunctival and corneal surface. Transplantation of amniontic membrane]. Ophthalmologe. 1998;95(12):80513. German.

7. Choi YS, Kim JY, Wee WR, Lee JH. Effect of the application of human amniotic membrane on rabbit corneal wound healing after excimer laser photorefractive keratectomy. Cornea. 1998;17(4):389-95.

8. Woo HM, Kim MS, Kweon OK, Kim DY, Nam TC, Kim JH. Effects of amniotic membrane on epithelial wound healing and stromal remodeling after excimer laser keratectomy in rabbit cornea. Br J Ophthalmol. 2001;85(3):345-9.

9. Zhang Q, Shimoya K, Moriyama A, Yamanaka K, Nakajima A, Nobunaga T, et al. Production of secretory leukocyte protease inhibitor by human amniotic 
membranes and regulation of its concentration in amniotic fluid. Mol Hum Reprod. 2001;7(6):573-9.

10. Campbell S. 4D, or not 4D: that is the question. Ultrasound Obstet Gynecol. 2002;19(1):1-4.

11. Longaker MT, Chiu ES, Adzick NS, Stern M, Harrison MR, Stern R. Studies in fetal wound healing $\mathrm{V}$. A prolonged presence of hyaluronic acid characterizes fetal wound fluid. Ann Surg. 1991;213(4):292-6.

12. Longaker MT, Whitby DJ, Jennings RW, Duncan BW, Ferguson MW, Harrison MR, et al. Fetal diaphragmatic wound healing with scar formation. J Surg Res. 1991;50(4):375-85.

13. Herretes S, Suwan-Apichon O, Pirouzmanesh A, Reyes JM, Broman AT, Cano $\mathrm{M}$, et al. Use of topical human amniotic fluid in the treatment of acute ocular alkali injuries in mice. Am J Ophthalmol. 2006;142(2):271-8.

14. Lee AS, Kim JC. Effect of amniotic fluid in corneal sensitivity and nerve regeneration after excimer laser ablation. Cornea. 1996;15(5):517-24.

15. Underwood MA, Gilbert WM, Sherman MP. Amniotic fluid: not just fetal urine anymore. J Perinatol. 2005;25(5):341-8.

16. Cho CK, Shan SJ, Winsor EJ, Diamandis EP. Proteomics analysis of human amniotic fluid. Mol Cell Proteomics. 2007;6(8):1406-15.

17. Modena AB, Fiena S. Amniotic fluid dynamics. Acta Biomed. 2004;75(Suppl 1): 11-13.

18. Lang AK, Searle RF. The immunomodulatory activity of human amniotic fluid can be correlated with transforming growth factor-beta 1 and beta 2 activity. Clin Exp Immunol. 1994;97(1):158-63.

19. Kasaian MT, Neet KE. Nerve growth factor in human amniotic and cerebrospinal fluid. Biofactors. 1989;2(2):99-104.

20. Fukuda H, Masuzaki H, Ishimaru T. Interleukin-6 and interleukin-1 receptor antagonist in amniotic fluid and cord blood in patients with pre-term, premature rupture of the membranes. Int J Gynecol Obstet. 2002;77(2):123-9.

21. Dudley DJ, Hunter C, Mitchell MD, Varner MW. Amniotic fluid interleukin10(IL-10) concentrations during pregnancy and with labor. J Reprod Immunol. 1997;33(2):147-56.

22. Karaçal N, Kosucu P, Cobanglu U, Kutlu N. Effect of human amniotic fluid on bone healing. J Surg Res. 2005;129(2):283-7.

23. Longaker MT, Adzick NS, Hall JL, Stair SE, Crombleholme TM, Duncan BW, et al. Studies in fetal wound healing, VII. Fetal wound healing may be modulated by hyaluronic acid stimulating activity in amniotic fluid. J Pediatr Surg. 1990;25(4):430-3.

24. Ozgenel GY, Filiz G. Effects of human amniotic fluid on peripheral nerve scarring and regeneration in rats. J Neurosurg. 2003;98(2):371-7.
25. Gao X, Devoe LD, Given KS. Effects of amniotic fluid on proteases: a possible role of amniotic fluid in fetal wound healing. Ann Plastic Surg. 1994;33(2): 128-34; discussion 134-5.

26. Adzick NS, Lorenz HP. Cells, matrix, growth factors, and the surgeon; the biology of scarless fetal wound repair. Ann Surg. 1994;220(1):10-8.

27. Kerimoglu S, Livaoglu M, Sönmez B, Yulug E, Aynac1 O, Topbas M, et al. Effects of human amniotic fluid on fracture healing in rat tibia. J Surg Res. 2008; [In press].

28. Ozgenel GY, Filiz G, Ozcan M. Effects of human amniotic fluid on cartilage regeneration from free perichondrial grafts in rabbits. Br J Plast Surg. 2004; 57(5):423-8.

29. Ozgenel GY. The influence of human amniotic fluid on the potential of rabbit ear perichondrial flaps to form cartilage tissue. Br J Plast Surg. 2002;55(3):246-50.

30. Ozgenel GY, Samli B, Ozcan M. Effects of human amniotic fluid on peritendinous adhesion formation and tendon healing after flexor tendon surgery in rabbits. J Hand Surg (Am). 2001;26(2):332-9.

31. Longaker MT, Chiu ES, Harrison MR, Cirombleholme TM, Langer JC, Duncan BW, et al. Studies in fetal wound healing: IV. Hyaluronic acidstimulating activity distinguishes fetal wound fluids from adult wound fluid. Ann Surg. 1989;210(5):667-72.

32. Kasper K, Frank B, Geerling G, Meinhardt B, Hartwig D. Amniotic fluid eyedrops versus serum eyedrops - an in vitro study [abstract]. Invest Ophthalmol Vis Sci. 2008. [In press].

33. Castro-Combs J, Noguera G, Cano M, Yew M, Gehlbach PL, Palmer J, et al. Corneal wound healing is modulated by topical application of amniotic fluid in an ex vivo organ culture model. Exp Eye Res 2008;87(1):56-63.

34. Siegel N, Rosner M, Hanneder M, Valli A, Hengstschlager M. Stem cells in amniotic fluid as new tools to study human genetic diseases. Stem Cell Rev. 2007;3(4):256-64.

35. Yan WH, Lin, A, Chen XJ, Dai MZ, Xu HH, Chen BG, et al. Immunological aspects of human amniotic fluid cells: implication for normal pregnancy. Cell Biol Int. 2008;32(1):93-9.

36. In 't Anker PS, Scherjon AS, Kleijburg-van der Keus C, Noort WA, Claas FH, Willemze R, et al. Amniotic fluid as a novel source of mesenchymal stem cells for therapeutic transplantation. Blood. 2003;102(4):1548-9.

37. De Coppi P, Bartsch G, Siddiqui MM, Xu T, Santos CC, Perin L, et al. Isolation of amniotic fluid stem cell lines with potential for therapy. Nat Biotechnol. 2007;25(1):100-6.

38. Fernandes M, Sridhar MS, Sangwan VS, Rao GN. Amniotic membrane transplantation for ocular surface reconstruction. Cornea. 2005;24(6):643-53. 